\title{
Biodegradable Harmonophores for Targeted High-Resolution In Vivo Tumor Imaging
}

Ali Yasin Sonay, Konstantinos Kalyviotis, Sine Yaganoglu, Aysen Unsal, Martina Konantz, Claire Teulon, Ingo Lieberwirth, Sandro Sieber, Shuai Jiang, Shahed Behzadi, Daniel Crespy, Katharina Landfester, Sylvie Roke, Claudia Lengerke, and Periklis Pantazis*

Cite This: ACS Nano 2021, 15, 4144-4154

Read Online

ACCESS | Llll Metrics \& More | 回 Article Recommendations ｜ sl Supporting Information

ABSTRACT: Optical imaging probes have played a major role in detecting and monitoring a variety of diseases. In particular, nonlinear optical imaging probes, such as second harmonic generating (SHG) nanoprobes, hold great promise as clinical contrast agents, as they can be imaged with little background signal and unmatched long-term photostability. As their chemical composition often includes transition metals, the use of inorganic SHG nanoprobes can raise long-term health concerns. Ideally, contrast agents for biomedical applications should be degraded in vivo without any long-term toxicological consequences to the organism. Here, we developed biodegradable harmonophores (bioharmonophores) that consist of

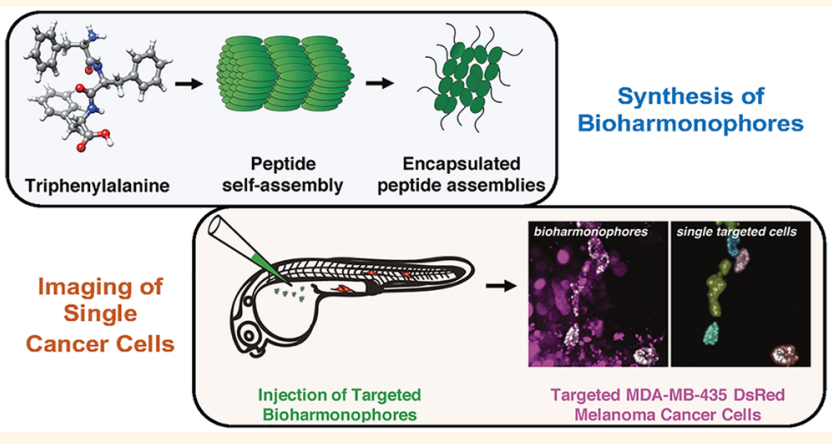
polymer-encapsulated, self-assembling peptides that generate a strong SHG signal. When functionalized with tumor cell surface markers, these reporters can target single cancer cells with high detection sensitivity in zebrafish embryos in vivo. Thus, bioharmonophores will enable an innovative approach to cancer treatment using targeted high-resolution optical imaging for diagnostics and therapy.

KEYWORDS: self-assembly, ferroelectric, biodegradable, second harmonic generation, imaging

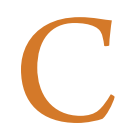

linical and preclinical imaging holds great potential in mapping disease progression and can provide diagnostic information ${ }^{1}$ that may guide the choice of treatment strategies for disease. ${ }^{2,3}$ Optical techniques using bioluminescent and fluorescent probes have emerged as promising modalities for molecular imaging in disease and therapy due to their ease of use and improved cellular resolution, capable of distinguishing boundaries between malignant and normal tissue. ${ }^{4}$ A key challenge for optical imaging probes and instrumentation, particularly those aimed at eventual clinical applications, is to overcome the problem of autofluorescence, which often result in a low signal-to-noise ratio (SNR). ${ }^{5}$ The relatively poor photostability of most imaging probes poses another challenge to provide reliable and sensitive imaging of tumors.

Previously, we introduced inorganic second harmonic generating (SHG) nanocrystals, SHG nanoprobes, ${ }^{6}$ as a class of imaging probes that can be used for in vivo imaging. ${ }^{7}$ Given that SHG imaging employs near-infrared (NIR) incident light for contrast generation, SHG nanoprobes can be utilized for deep tissue imaging. ${ }^{8,9}$ Unlike commonly used fluorescent probes, SHG nanoprobes neither bleach nor blink, and their signal does not saturate with increasing illumination intensity, ensuring high probe sensitivity. ${ }^{10}$ Since their signal profile is very narrow, they can be imaged with high SNR by excluding the broad emission of typical autofluorescence background. ${ }^{6,11}$ Robust functionalization allows targeting to a wide variety of cells and proteins of interest, ${ }^{12}$ allowing these imaging probes to be promising tools for both clinical and preclinical imaging applications. ${ }^{13}$ Despite these advantages, the chemical structure of inorganic SHG nanoprobes makes them stable in the body, which may cause concerns for the long-term health of an organism that has been imaged with these reporters. ${ }^{14}$

To create a foundation for safe SHG nanoprobe-based clinical imaging, we set out to generate a nanoprobe that consists of biodegradable materials, capable of generating sufficient SHG signal that can be detected with high SNR. Our efforts were guided by the observation that peptides with a

Received: December 19, 2020

Accepted: February 5, 2021

Published: February 25, 2021 
a
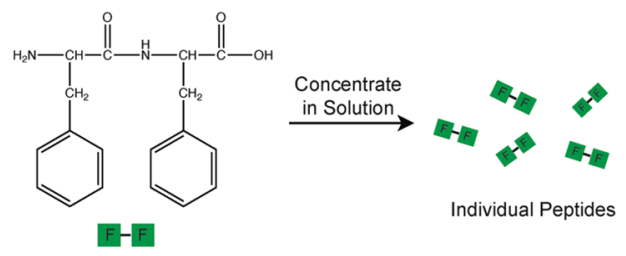

$\stackrel{\text { Self-assembly }}{\longrightarrow}$

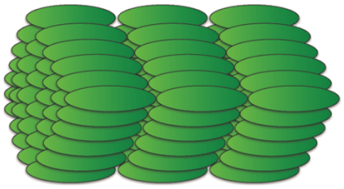

Individual Peptides

Aggregated Peptide

Nanotubes

b

Continuous Phase (Water and SDS)

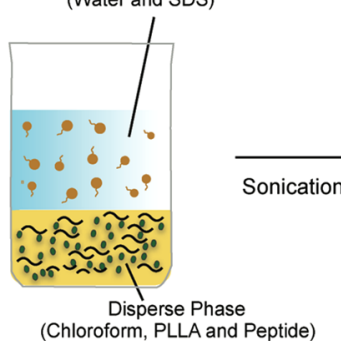

Chloroform Droplet

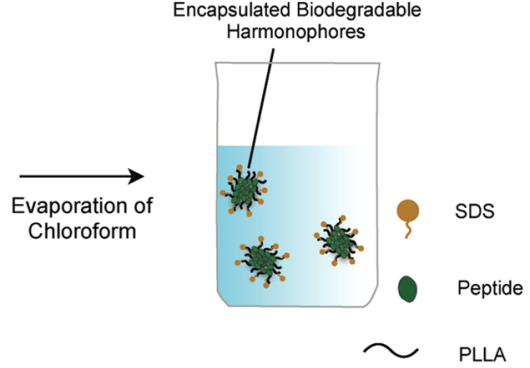

(Chloroform, PLLA and Peptide)

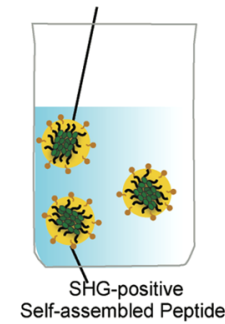

C

d

e
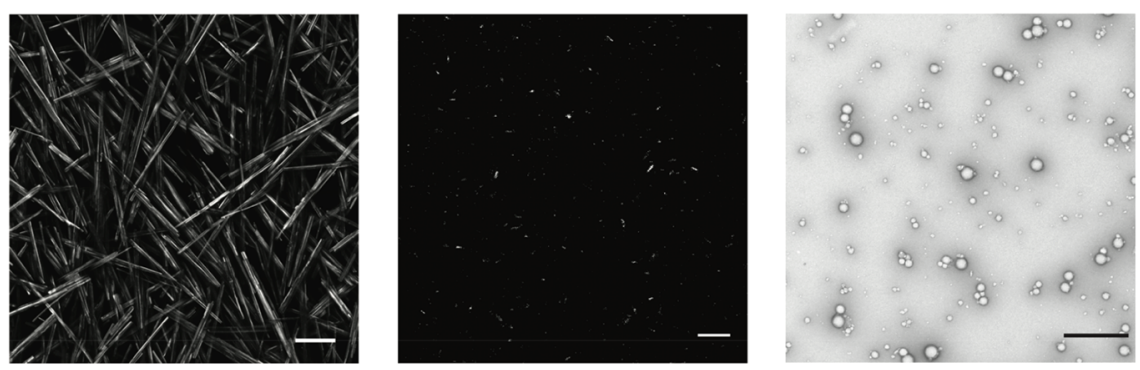

f

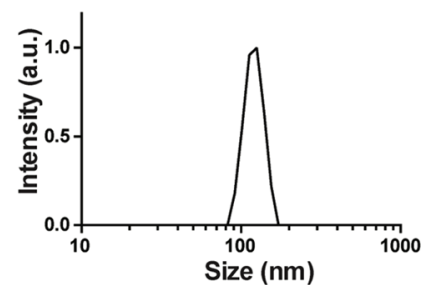

Figure 1. Synthesis and analysis of bioharmonophores. (a) Schematic of the self-assembling reaction of diphenylalanine peptides (FF) into large-scale nanotube structures from a concentrated solution. (b) Schematic of the emulsion-solvent evaporation method for the synthesis of bioharmonophores. Self-assembling peptides are dissolved in chloroform along with biodegradable poly(L-lactic acid) (PLLA) and emulsified with the surfactant sodium dodecyl sulfate (SDS) using sonication, followed by evaporation of chloroform. (c) SHG signal from diphenylalanine peptide nanotubes aggregated on top of the imaging chamber. Peptide nanotubes were illuminated with a $850 \mathrm{~nm}$ pulsed laser. Image composite of multiple stitched images. (d) SHG signal from encapsulated triphenylalanine peptide (FFF) bioharmonophores immobilized in $1 \%$ low melting agarose illuminated with $850 \mathrm{~nm}$ pulsed laser. (e) TEM image of synthesized FFF-based bioharmonophores showing uniform spherical nanoparticles. (f) DLS data showing the size distribution of synthesized bioharmonophores. Scale bar, $100 \mu \mathrm{m}$ (c); $10 \mu \mathrm{m}(\mathrm{d}) ; 500 \mathrm{~nm}(\mathrm{e})$.

variable number of amino acid units can self-assemble into large, solid nanostructures of different morphologies and symmetries (Figure 1a). ${ }^{15}$ It has been previously shown that such structures can be ferroelectric and give nonlinear optical contrast such as SHG (Figure 1c). ${ }^{16,17}$ Since these peptides self-assemble in solution in an inherently sequential process, they form nanostructures in the micrometer range that are difficult to control in size and cannot be readily utilized for in vitro and in vivo imaging. Given that the SHG signal output is dependent on the size of the assembly and significantly decreases at the nanoscale, we set out to develop a synthesis method that would miniaturize these peptides assemblies and control their size into nanoparticles. Importantly, miniaturized peptide assemblies should not lose their crystalline structure and SHG signal, generating imaging agents that can be degraded over time without harming the body.

\section{RESULTS AND DISCUSSION}

Synthesis of Bioharmonophores. To render these nanostructures suitable for biological applications, we evaluated methods for the encapsulation of self-assembling peptides in order (i) to hinder their macroscopic aggregation by confining their self-assembly in nanodroplets without affecting their ability to generate a strong SHG signal and (ii) to generate a nanoparticle that can be further functionalized without influencing the peptide assembly. To this end, we subjected several peptides that have been reported to selfassemble into complex nanostructures to the emulsion-solvent evaporation method, ${ }^{18}$ a widely used procedure for the 
a
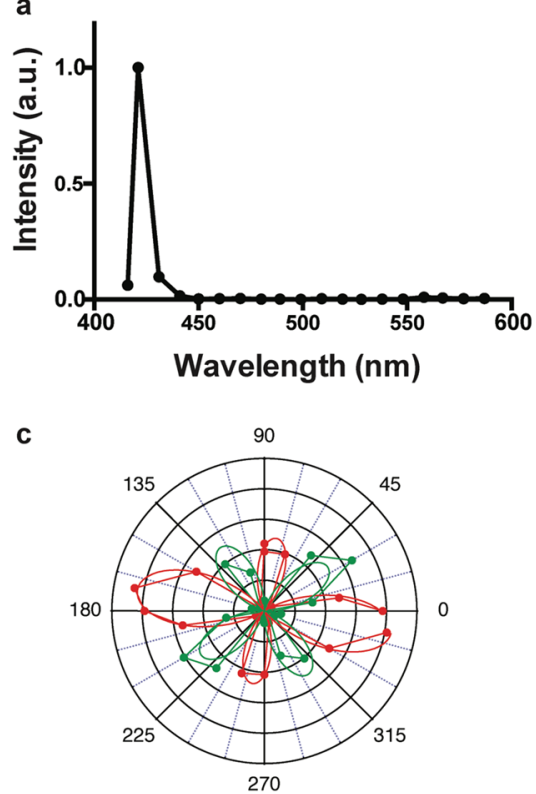

e

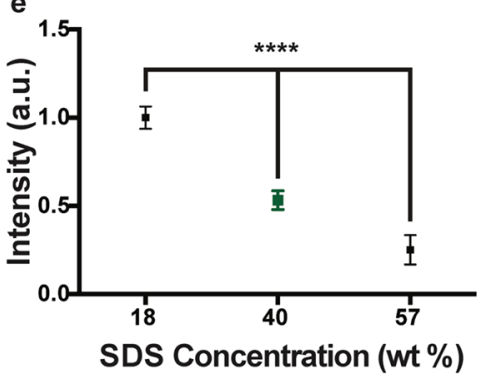

b

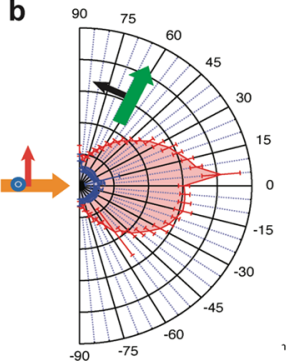

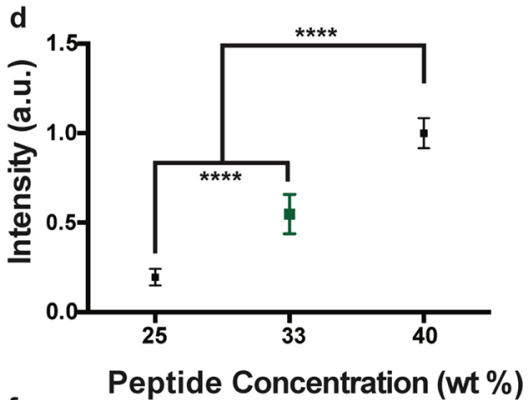

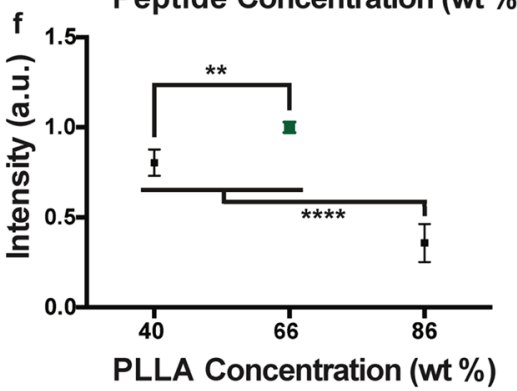

Figure 2. Optical characterization of bioharmonophores and analysis of parameters influencing bioharmonophore formation. (a) Normalized SHG signal spectrum of FFF-based bioharmonophores (signal ranging from 400 to $600 \mathrm{~nm}$ ) illuminated with $850 \mathrm{~nm}$ pulsed laser. The characteristic SHG peak is centered around $425 \mathrm{~nm}$. (b) SHG emission pattern of Triphenylalanine based bioharmonophores. Orange arrow indicates excitation beam direction. Green arrow shows SHG collection direction, which rotates between $-90^{\circ}$ and $+90^{\circ}$. The detected polarization is in the beams plane ( $P$, black arrow). The red pattern shows PPP polarization configuration (excitation and detection polarizations in the plane of the beams), and the blue pattern shows PSS (excitation with a perpendicular polarization). (c) SHG intensity vs incident polarization angle for a bioharmonophore, highlighted by the solid white circle in Supplementary Figure 5. Red color shows detection along the $\mathrm{X}$ axis while green color shows detection along the $\mathrm{Y}$ axis. The experimental curve is a dotted line, the corresponding fitted curve, assuming $C_{2}$ symmetry, is a solid line. (d) Influence of using different amounts of FFF peptide during bioharmonophore production on the SHG signal intensity. The optimal condition ( $33 \mathrm{wt} \%)$ is marked in green. The use of higher FFF peptide amount leads to aggregates $(n=5)$. (e) Influence of SDS concentration (wt \% of disperse phase) on SHG intensity of generated bioharmonophores. The optimal condition ( $40 \mathrm{wt} \%$ SDS) with high bioharmonophore stability and less aggregation is marked in green $(n=5)$. (f) Influence of using different amounts of PLLA during bioharmonophore production on the SHG intensity of the generated bioharmonophores. The optimal condition (66 wt \% PLLA) is marked in green $(n=5)$. Mean \pm s.d. ****, $P<0.0001, * *, P<0.005, *, P<0.05$ (ordinary one-way ANOVA with Tukey's multiple comparisons).

fabrication of monolithic and core-shell nanoparticles (Figure $1 b$, see the Experimental Methods).

We identified three peptides with different self-assembling properties (pentaalanine, ${ }^{19}$ trileucine, ${ }^{20}$ and triphenylalanine $\mathrm{e}^{21}$ ) that could generate a detectable SHG signal when encapsulated in the biodegradable polymer (Figure 1d and Supplementary Figure 1). Transmission electron microscopy (TEM) analysis of the predominantly spherical nanoparticles, hereinafter referred to as bioharmonophores, revealed a diameter ranging from 50 to $150 \mathrm{~nm}$ (Supplementary Figure 2), which was confirmed by dynamic light scattering (DLS) measurements (Figure 1e,f).

SHG signal from bioharmonophores can stem from (i) the bulk of the self-assembling peptides that form noncentrosymmetric crystalline structures or (ii) the surface of the bioharmonophores where there is no inversion symmetry. To ascertain that the SHG signal originates from the crystalline peptide core, we performed X-ray diffraction (XRD) analysis of bioharmonophores with different peptide contents. In all cases, the peptides showed a high degree of internal order with distinct diffraction patterns associated with their individual crystalline phases and self-assembling behavior (Supplementary Figure $3 a-d)$.

Optical Characterization of Bioharmonophores. Because bioharmonophores based on triphenylalanine (FFF) peptides yielded the strongest SHG signal compared to pentaalanine and trileucine, we subjected these bioharmonophores to detailed optical characterizations. The SHG signal of FFF-based bioharmonophores was spectrally well-defined (Figure 2a) and comparable with inorganic SHG nanoprobes (Supplementary Figure 4). Additionally, the SHG emission patterns of FFF-based bioharmonophores displayed a broad 
a

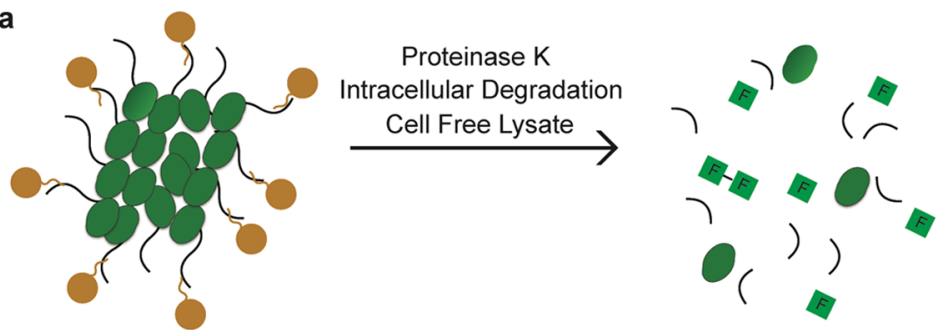

High SHG Signal

Low SHG Signal
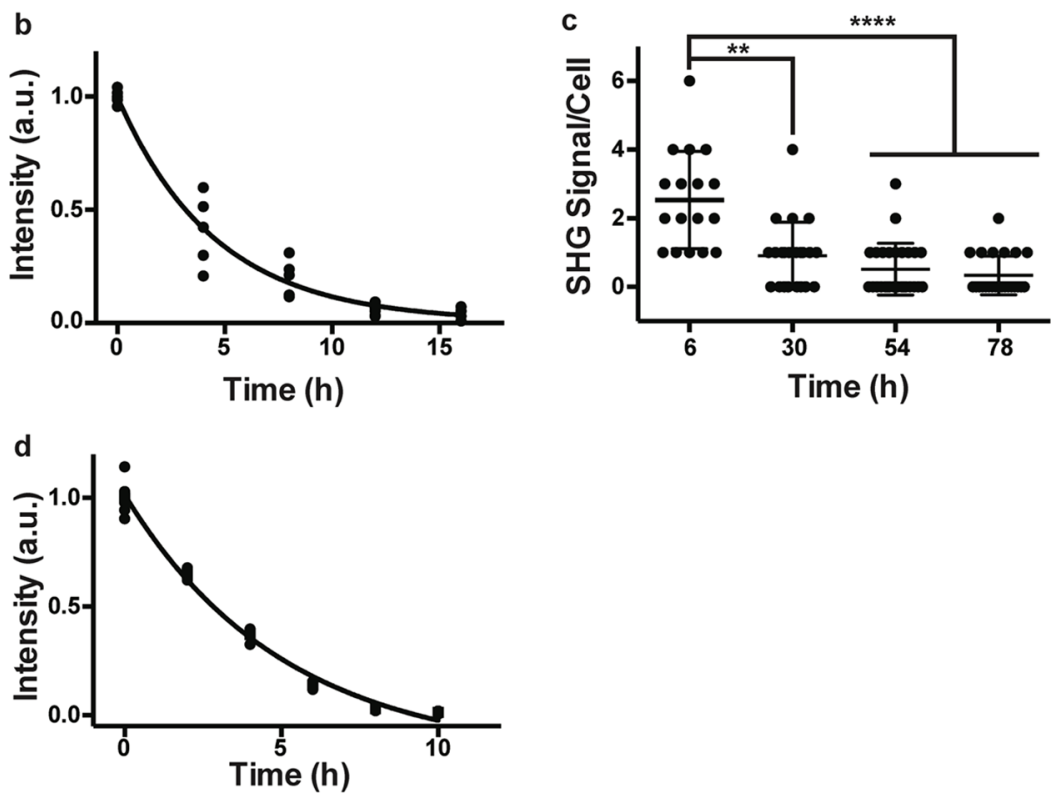

Figure 3. Bioharmonophores can be degraded by proteases, cells, and cell-free lysate systems. (a) Schematic showing different degradation methods utilized to assess biodegradability of the bioharmonophores. (b) Graph displaying the change of SHG signal intensity over time of bioharmonophores incubating with proteinase $\mathrm{K}(n=5)$. Mean values of data points were fitted for one-phase exponential decay. (c) Quantification of SHG signal/cell after overnight incubation of Tat-peptide functionalized bioharmonophores over time. SHG signal/cell is significantly reduced $30 \mathrm{~h}$ after reseeding. Mean \pm s.d. $* * *, P<0.0001, * *, P<0.005, *, P<0.05$ (nonparametric Kruskal-Wallis test with Dunn's post hoc multiple comparison). (d) Graph showing the loss of SHG signal intensity when bioharmonophores are subjected to the cell-free reticulate lysate degradation system $(n=5)$. Mean values of data points were fitted using a one phase exponential decay. Scale bar, $10 \mu \mathrm{m}$ (c); $10 \mu \mathrm{m}$ (d).

opening: one seemingly isotropic (Figure 2b, PSS polarization, blue data) and the other one displaying one lobe with a width of $60^{\circ}$ centered around the forward direction (Figure 2b, PPP polarization, red data). These results indicate that bioharmonophores emit an SHG signal in multiple directions, which can be detected in an epi-detection setup (i.e., illumination and collection of SHG signal using the same objective lens), unlike the predominantly forward-directed SHG signal of large protein arrays, such as collagen and myosin that require a trans-detection approach (i.e., illumination and SHG signal traverses the sample to reach the detector). ${ }^{10}$ This difference in detection geometry allows exclusion of a collagen-based SHG signal during imaging, leading to a high SNR bioharmonophore signal that can be distinguished from large-scale collagen arrays even in tissues with high SHG background. Moreover, the presence of a single lobe suggests that the observed SHG signal originates from the bulk ${ }^{22}$ of the bioharmonophores and not from its surface, as described by nonlinear light scattering theory. $^{22,23}$ The high intensity emission in the PPP polarization combination further indicates that a coherent response originates from a noncentrosymmetric crystalline bulk.
Because SHG involves only virtual energy transitions, bioharmonophores did not display blinking and remained stable over extended periods of illumination, and their SHG signal intensity rose quadratically when the laser intensity shone on them was linearly increased (Supplementary Figure $5 a, b)$. The measured polarimetric diagrams (Figure $2 \mathrm{c}$ and Supplementary Figure $5 c-f$ ) were consistent with the hypothesis that bioharmonophores have a self-assembling peptide core with a monoclinic (C2) symmetry. Indeed, the experimental curves were well fitted with the analytical expression calculated for this symmetry (see Supplementary Note 1). Taken together, bioharmonophores have the same photophysical advantages for biomedical imaging applications that have been previously described for inorganic SHG nanoprobes. ${ }^{6}$

To gain insight into the parameters influencing the bioharmonophore stability and signal intensity, we tested several reaction conditions to generate bioharmonophores. Given that the SHG signal originating from bioharmonophores is dependent on the amount of encapsulated peptide, we first tested whether varying the FFF peptide concentration during production would improve the SHG signal intensity of 
a
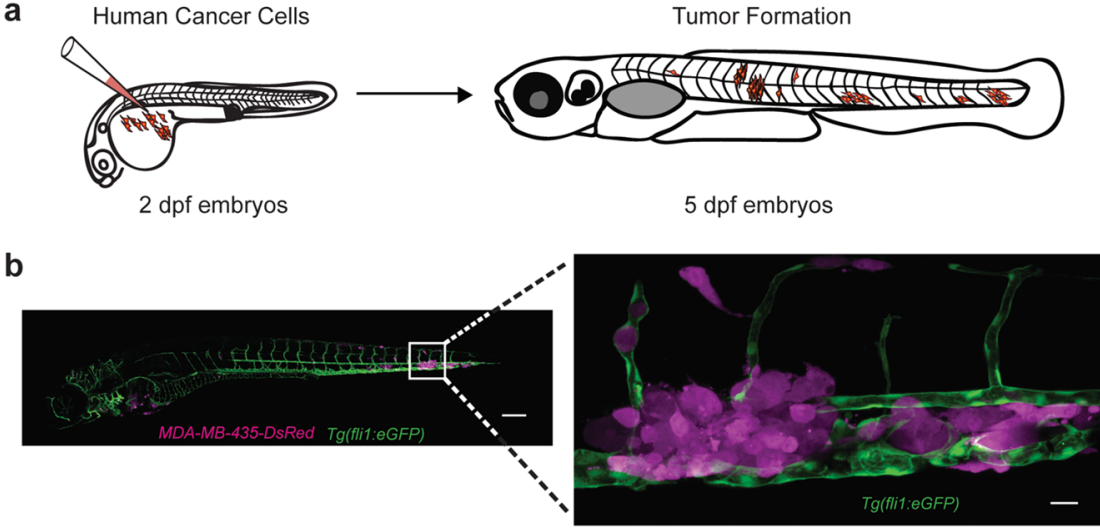

C
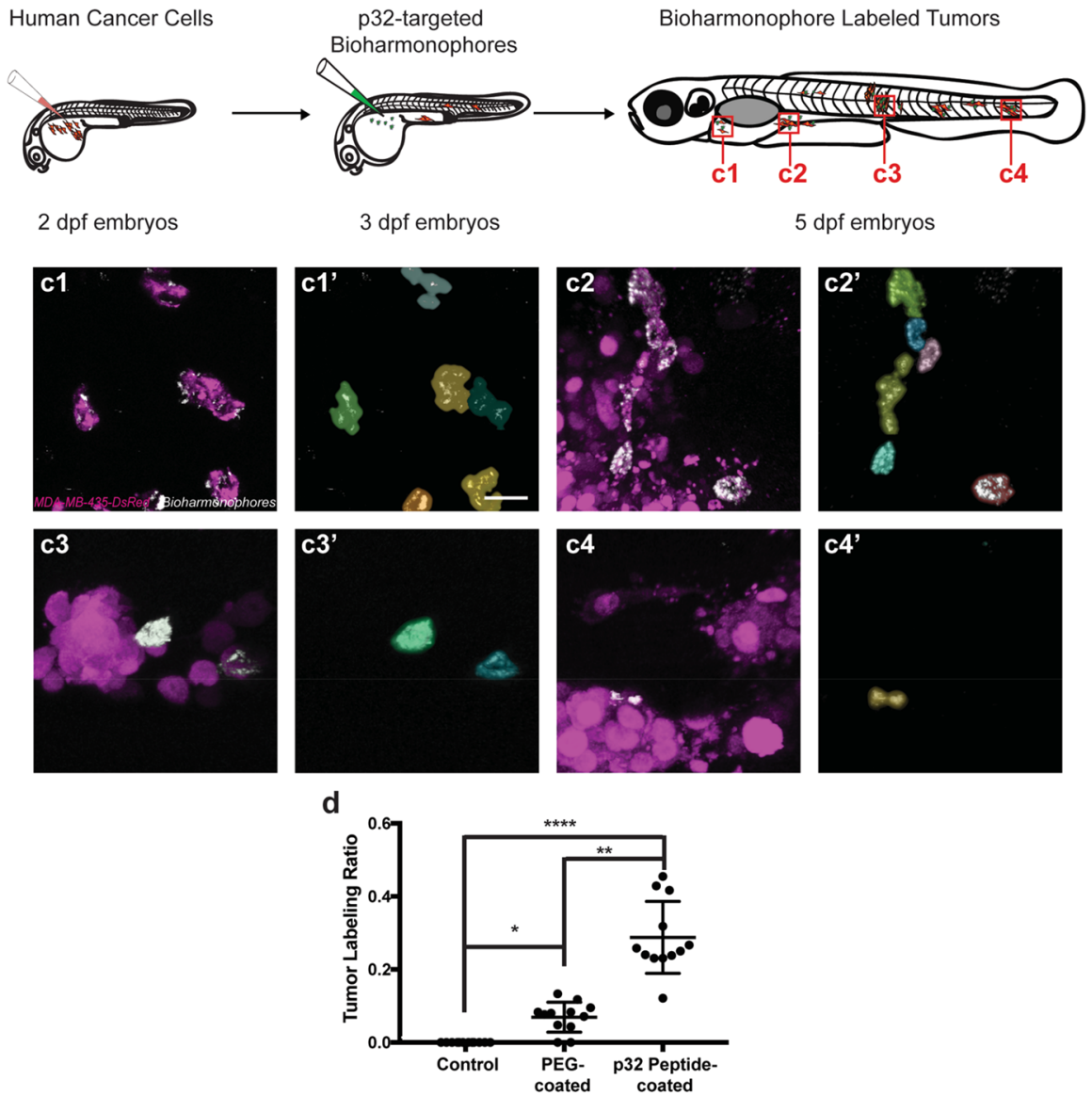

Figure 4. Bioharmonophores can be specifically targeted to single cancer cells in vivo. (a) Schematic showing the generation of a zebrafish cancer model by injecting MDA-MB-435-DsRed cancer cells into the Duct of Cuvier (DoC) at 2 dpf, resulting in tumors spread to multiple locations of the zebrafish body at $5 \mathrm{dpf}$. (b) Composite image of the cancer model (left) in a $5 \mathrm{dpf}$ old zebrafish embryo. Close-up image of one of the tumor sites (right) reveals DsRed-labeled tumors (magenta), adjacent to the eGFP-labeled vasculature (green). (c) Schematic showing cancer cell injection of $2 \mathrm{dpf}$ zebrafish embryos followed by bioharmonophore injection into DoC of $3 \mathrm{dpf}$ zebrafish embryos and subsequent fluorescence and SHG imaging at $5 \mathrm{dpf}$. Red rectangles labeled as c1-4 denote the regions of interest that are illustrated in more detail. Individual panels showing the images of labeled cancer cells with the details of bioharmonophore (white) labeling down to single cancer cells (magenta) in solid tumors (c1-4). Colored cell boundary reconstruction of targeted cancer cells using the bioharmonophore SHG signal $\left(\mathrm{cl}^{\prime}-4^{\prime}\right)$. Note that cellular bioharmonophore distribution can in most cases predict cell morphologies. Scale bar, left panel 200 $\mu \mathrm{m}$, right panel $20 \mu \mathrm{m}(\mathrm{b}) ; 15 \mu \mathrm{m}(\mathrm{c})$. (d) Quantification of the fraction of SHG-labeled tumors as the ratio of labeled tumors to all tumors in a given zebrafish embryo after PEG- and p32 peptide-coated bioharmonophore injection, respectively. Each data point signifies one zebrafish. Note that active targeting with p32-coated bioharmonophores significantly increases the labeling efficiency (approximately 4-fold). Mean \pm s.d. $* * *, P<0.0001$, **, $P=0.0063, *, P=0.0470$ (nonparametric Kruskal-Wallis test with Dunn's post hoc multiple comparison). $N=12$, pooled from 3 independent experiments.

generated bioharmonophores (Figure 2d and Supplementary Figure 6). We found that an amount of $15 \mathrm{mg}$ of FFF (33 wt $\%)$ peptide provided an optimal combination of intense SHG signal and bioharmonophore stability. Interestingly, while an FFF peptide amount of $20 \mathrm{mg}$ (40 wt \%) increased the overall SHG signal, it also led to bioharmonophore aggregation and 
decreased colloidal stability. Conversely, $10 \mathrm{mg}$ (25 wt \%) of FFF peptide generated little SHG signal.

Because surfactant concentration plays a crucial role in emulsification of chloroform droplets, ${ }^{18}$ we reasoned that altering the surfactant concentration during the preparation of bioharmonophores would have a profound effect on their stability and signal strength (Figure 2e and Supplementary Figure 7). Bioharmonophores emulsified in an aqueous solution with $0.3 \%$ sodium dodecyl sulfate (SDS) (40 wt \% of dispersed phase) yielded stable bioharmonophores with intense SHG signal, whereas compositions employing $0.1 \%$ SDS (18 wt \%) yielded aggregated nanoparticles. Increasing the SDS concentration to $0.6 \%$ (57 wt \%) diminished the SHG signal intensity, suggesting that the bioharmonophore size and hence the number of enclosed peptide molecules within each bioharmonophore is influenced by the concentration of surfactant.

Finally, we varied the polymer quantity that encapsulates and shields peptides from environmental changes and assessed its role in both SHG signal intensity and nanoparticle morphology (Figure 2f and Supplementary Figure 8). We identified that an amount of $30 \mathrm{mg}$ of poly(L-lactic acid) (PLLA) (66 wt \%) resulted in an optimal combination of intense SHG signal and bioharmonophore stability. A lower polymer amount of $10 \mathrm{mg}$ (40 wt \%) yielded a weaker SHG signal, whereas a higher polymer amount ( $90 \mathrm{mg}$, $86 \mathrm{wt} \%)$ led to elongated bioharmonophore morphologies. Taken together, we identified optimal experimental conditions to generate bioharmonophores providing a high SNR along with an excellent stability and size distribution for biological applications.

Biodegradation of Bioharmonophores. Clinical imaging probes that are biodegradable provide the significant advantage of being able to be broken down in the body and removed after they have served their function. To demonstrate that bioharmonophores are indeed biodegradable, we utilized the highly effective serine protease, proteinase $\mathrm{K}$, which exhibits a broad cleavage specificity. ${ }^{24}$ We incubated bioharmonophores with a proteinase $\mathrm{K}$ concentration that is routinely used for dissolving tissue structures ${ }^{25}$ and probed the extent of degradation by monitoring the SHG signal at different time intervals (Figure 3a). We observed a decrease of SHG signal within $2 \mathrm{~h}$ of protease incubation. After $10 \mathrm{~h}$, the SHG signal disappeared and the turbid bioharmonophore suspension became transparent (Figure $3 \mathrm{~b}$ and Supplementary Figure 9), indicating a successful biodegradation of the bioharmonophore. When characterized by TEM imaging, the structural integrity of the bioharmonophore coat started to disintegrate within $4 \mathrm{~h}$ of Proteinase $\mathrm{K}$ treatment (Supplementary Figure 10).

To evaluate bioharmonophore degradation under physiological conditions (Figure 3c), we functionalized bioharmonophores with Tat-derived cell penetrating peptides ${ }^{26}$ using bioorthogonal click chemistry (Supplementary Figure 11) and incubated them with a model cancer cell line (see below) overnight. Adherent cells were then detached by trypsinization and centrifuged to remove excess bioharmonophores that did not enter the cancer cells. Following this procedure, cells were reseeded and fixed at specific time periods to monitor bioharmonophore degradation (i.e., the intracellular presence of SHG signal per cell) using nonlinear optical imaging. Thirty hours after cell reseeding, a pronounced decrease of intracellular SHG signal per cell was noticeable (Figure 3c). As bioharmonophores displayed long-term photostability even at low $\mathrm{pH}$ values (Supplementary Figure 12), the drop of signal was not due to their potential accumulation in acidic endolysosomal compartments over time. Immunostaining of uptaken bioharmonophores indeed revealed that bright SHG signal still persisted in lysosome-associated membrane protein 2 (LAMP2)-positive endocytic compartments prior to degradation (Supplementary Figure 13). In order to show that bioharmonophores can be degraded using intracellular proteolytic degradation, we tested whether the bioharmonophores could be degraded using a cell-free lysate system based on an established cell-free degradation assay. ${ }^{27} \mathrm{We}$ also observed reduced SHG signal, indicating that intracellular enzymatic degradation of bioharmonophores might account for the signal loss (Figure 3d and Supplementary Figure 14). Importantly, bioharmonophores did not exhibit any short-term toxicity in vitro and in vivo (Supplementary Figure 15) and did not induce protein aggregation (Supplementary Figure 16), ${ }^{28}$ rendering them safe imaging probes.

In Vivo Cancer Imaging. Among various diagnostic applications, bioharmonophores could be ideal imaging probes for single-cell cancer detection due to their high SNR and photostability, which other intravital imaging modalities cannot achieve. ${ }^{2}$ To demonstrate the optical features of bioharmonophores for cancer targeting and imaging, we employed xenograft zebrafish cancer models, which offer speed, cellular resolution, and the ability to perform large numbers of transplants for obtaining valuable information about several cancer types. ${ }^{29,30}$

To generate a highly aggressive cancer model that can be tracked over time, we injected a DsRed-expressing metastatic human melanoma cells (MDA-MB-435-DsRed) into the Duct of Cuvier (DoC) of zebrafish embryos at $2 \mathrm{dpf}$ (days post fertilization) (Figure 4a). ${ }^{29,30}$ By 3 days after the injection, the resulting tumors spread to various locations in the body and were found next to blood vessels, which likely support the tumors with nutrients (Figure $4 \mathrm{~b}$ ). ${ }^{31}$

To demonstrate the specificity and efficiency of bioharmonophores as contrast agents that can accomplish resolution down to the single cell in vivo, we targeted bioharmonophores to tumor sites by taking advantage of the surface protein p32/ $\mathrm{gClqR}$ as a characteristic molecular marker for MDA-MB-435DsRed cells. ${ }^{32}$ To this end, we functionalized bioharmonophores with a p32 targeting peptide, injected them into the DoC of zebrafish embryos at $3 \mathrm{dpf}, 1$ day after the embryos were injected with MDA-MB-435-DsRed cancer cells (Figure $4 c)$, and assessed colocalization between cancer cells and bioharmonophore signal at $5 \mathrm{dpf}$ (see Supplementary Note 2). In the absence of tumors, functionalized bioharmonophores did not cause clustering at the site of injection and were localized at different parts of injected zebrafish embryos (Supplementary Figure $17 \mathrm{~d}-\mathrm{i}$ ), indicating good biodistribution. Without bioharmonophore injection, zebrafish as well as tumor sites did not produce any SHG background signal (Supplementary Figures $17 \mathrm{a}-\mathrm{c}$ and $18 \mathrm{a}-\mathrm{c}$ ) with the exception of minimal endogenous SHG signal localized at the zebrafish tail, ${ }^{33}$ which was excluded from assessing specificity of tumor targeting (Supplementary Figure 19). In the case of passive targeting, zebrafish injected with PEG-coated bioharmonophores revealed limited tumor labeling (Supplementary Figure 18d-f), stemming from leaky blood vessels and enhanced permeability and retention effect (EPR). ${ }^{34}$ In contrast, we observed an increased accumulation of p32 peptide-targeted 
bioharmonophores within individual cancer cells at tumor sites throughout the zebrafish embryos (Figure $4 c 1-c 4^{\prime}$ ), indicating that the tumor-labeling specificity and efficiency are highly dependent on the p32 targeting peptide. While p32 peptidetargeted bioharmonophores can extravasate to different tumor sites, not all the cancer cells were labeled (Figure 4c). This observation is potentially due to limited accessibility within densely packed solid tumors ${ }^{35}$ and the continued proliferation and metastasis of cancer cells between bioharmonophore administration and imaging (see Supplementary Note 2).

In order to determine the extent of labeling of targeted bioharmonophores in the xenograft zebrafish cancer model, we measured the colocalization of cancer cells with bioharmonophores at each tumor site for noninjected zebrafish as well as for zebrafish that were injected with p32 peptide-targeted and PEG-coated bioharmonophores, respectively (see Supplementary Note 2 and Figure 4c,d). The number of tumors were not significantly different between data sets (Supplementary Figure $20)$. The zebrafish cancer model injected with p32 peptidetargeted bioharmonophores had a significantly higher fraction of labeled tumors compared with noninjected and PEG-coated bioharmonophores (Figure $4 \mathrm{~d}$ ) due to our active targeting strategy. Overall, these results demonstrate that bioharmonophores exhibit high SNR and outstanding photostability for efficient labeling of individual cancer cells at multiple tumor sites in vivo.

\section{CONCLUSIONS}

In summary, we introduced bioharmonophores as a class of imaging probes that retain all the photophysical advantages of previously introduced inorganic SHG nanoprobes. Because bioharmonophores consist of a biodegradable peptide core and a polymer shell, they can be metabolized within cells. Our experiments indicated a degradation time between 24 and $48 \mathrm{~h}$ after injection, ${ }^{36-38}$ which is similar to existing nanoparticle based and molecular imaging agents. Their relative stability before finding their target and degradation upon cellular entry could render bioharmonophores ideal contrast agent for clinical imaging applications. The straightforward implementation of robust functionalization strategies and a sufficiently high metabolic stability in vivo allowed us to target bioharmonophores with high detection sensitivity to individual tumor cells in live zebrafish embryos. With the recent development of nonlinear microendoscopes, ${ }^{39,40}$ bioharmonophores have the potential to emerge as superior contrast agents during image-guided surgery to help surgeons perform safer and highly precise tumor removal procedures. Moreover, their ability to target single cells could be exploited for detecting cancer stem cells, a subpopulation of cells responsible for tumorigenicity, invasion, and metastasis. ${ }^{41}$ Once successfully identified, the nonlinear signal of bioharmonophores could be used for light induced drug delivery or photodynamic therapy. $^{42}$ By employing pulsed lasers in the infrared wavelength range that permit deep tissue penetration, targeted bioharmonophore signal could trigger highly localized cancer stem cell death. ${ }^{43}$ Finally, as the SHG signal intensity of bioharmonophore relies on the self-assembly behavior of each peptide, ${ }^{44}$ we anticipate that a screen for alternative peptide sequences may yield even brighter bioharmonophores that will potentially permit diagnosis with deep-tissue single-molecule detection sensitivity.

\section{EXPERIMENTAL METHODS}

Formation of Large-Scale Peptide Nanotubes. Diphenylalanine (FF) and triphenylalanine (FFF) (Bachem) peptide assemblies were prepared as previously described. ${ }^{45}$ Briefly, peptides were freshly dissolved in hexafluoroisopropanol (Sigma) at $100 \mathrm{mg} / \mathrm{mL}$ concentration prior to experiments and diluted to $5 \mathrm{mg} / \mathrm{mL}$ final concentration in deionized water.

Encapsulation of SHG-Active Peptide Assemblies. For the evaluation of different peptides and their SHG capabilities, $30 \mathrm{mg}$ of PLLA was dissolved in $3 \mathrm{~mL}$ of chloroform (Sigma) along with $15 \mathrm{mg}$ of triphenylalanine, $30 \mathrm{mg}$ of pentalalanine (Bachem), and $30 \mathrm{mg}$ of trileucine (Sigma) peptides in separate glass vials. The resulting suspension was mixed with aqueous SDS (Sigma) solution with a final $0.3 \%$ SDS concentration. A macroemulsion was obtained by stirring the samples at $1000 \mathrm{rpm}$ for $1 \mathrm{~h}$. Afterward, the samples were sonicated (Branson Sonifier) with a 1.5 in. tip at $70 \%$ power in a pulsed mode ( $30 \mathrm{~s}$ ON and $10 \mathrm{~s}$ OFF) for 2 min under ice cooling. The chloroform was evaporated from the obtained emulsions by stirring the samples at $500 \mathrm{rpm}$ at $40{ }^{\circ} \mathrm{C}$ overnight. For the remaining experiments with triphenylalanine peptide containing bioharmonophores, the same protocol was followed unless stated otherwise. For probing the optimal conditions for nanoparticle formation, FFF peptide, PLLA, and SDS concentrations were varied as described in Supplementary Figures 6-8.

Characterization of Encapsulated SHG-Active Peptide Assemblies. Produced samples were characterized using dynamic light scattering. Nanoparticle morphology, aggregation tendency, and the SHG signal intensity were evaluated using nonlinear microscopy. XRD patterns were obtained using a PANalytical X'PERT Pro powder diffractometer in Bragg-Brentano geometry and with $\mathrm{Cu}$ K- $\alpha 1$ radiation in grazing incidence geometry between $2-60$ using a step size of 0.0167 . The samples were air-dried on silicon single crystals, and four identical scans were obtained from each sample and summed up.

SHG Polarimetry. The SHG polarimetry was performed on a wide-field SHG microscope (see the Supporting Information). A 1030 $\mathrm{nm}$ laser, pulse width $190 \mathrm{fs}$, and $200 \mathrm{kHz}$ repetition rate (Pharos, Light Conversion), delivered $36 \mathrm{~mW}$ on the sample over a $150 \mu \mathrm{m}$ fwhm diameter field-of-view $\left(1 \mathrm{~mJ} \cdot \mathrm{cm}^{-2}\right)$. Two non-colinear beams are incident on the sample, with a $30^{\circ}$ opening angle. The SHG signal was detected in the phase matching direction (transmission). The image was recorded with an electron-multiplying intensified chargecoupled device (EM-ICCD) camera. Nonlinear polarimetry was performed by controlling and analyzing the polarization state of the illuminating and emitted beams. A polarization state generator, comprising a half- and a quarter-wave plate, was used. The polarization state of the emitted light was analyzed with a half-wave plate placed in the emission path, followed by a polarizing beam splitter.

Second-Harmonic Spectroscopy Patterns. SHG emission pattern measurement was performed on a custom-build setup for this purpose (see the Supporting Information). Excitation was performed with a $1030 \mathrm{~nm}$ laser, pulse width $190 \mathrm{fs}$, and $200 \mathrm{kHz}$ repetition rate, which delivered $60 \mathrm{~mW}$ on the sample, a cylindrical cuvette containing the solution, over a $36 \mu \mathrm{m}$ focal spot $(30 \mathrm{~mJ}$. $\mathrm{cm}^{-2}$ ). The signal was detected with a rotating PMT and a filter (515 +10 , Chroma) at angles between -90 and 90 . Both incident and detection polarizations was controlled.

Stability of Biodegradable Bioharmonophores at Different $\mathrm{pH}$ Values. To evaluate how different $\mathrm{pH}$ values might influence the PLLA-coated peptide assemblies and their signal intensity, synthesized bioharmonophores were centrifuged for $3 \mathrm{~min}$ at $13500 \mathrm{rpm}$ and resuspended in citric acid $/ \mathrm{Na}_{2} \mathrm{HPO}_{4}$ buffer ranging from 4 to $7 \mathrm{pH}$ values. The bioharmonophores were incubated for $72 \mathrm{~h}$ in the buffers containing $1 \%$ Tween 80 to prevent aggregation, and the signal intensity was monitored using nonlinear microscopy.

Biodegradation of Bioharmonophores in Vitro. Bioharmonophores were centrifuged for $3 \mathrm{~min}$ at $13500 \mathrm{rpm}$ and resuspended in $1 \%$ Tween 80 in 1X PBS. In order to assess proteinase K (Sigma) degradation, $1 \mathrm{~mL}$ of bioharmonophore suspension was incubated 
with $100 \mu \mathrm{g} / \mathrm{mL}$ final proteinase $\mathrm{K}$ concentration at $37{ }^{\circ} \mathrm{C}$ and the SHG signal intensity was measured every $2 \mathrm{~h}$. Similarly, in order to assess how bioharmonophores were degraded using cellular content, an ex vivo biodegradation protocol was adapted based on the Rabbit Reticulocyte Lysate system (Promega). In a typical setup, $1 \mathrm{~mL}$ of bioharmonophore in $1 \%$ Tween 80 in 1X PBS was mixed with $25 \mathrm{mM}$ phosphocreatine (Sigma), $10 \mu \mathrm{g} / \mathrm{mL}$ phosphocreatine kinase (Sigma), $1 \mathrm{mM}$ ATP (Sigma), and $50 \mu \mathrm{L}$ Rabbit Reticulocyte Lysate. The mixture was incubated at $37^{\circ} \mathrm{C}$, and the SHG signal intensity was monitored every $2 \mathrm{~h}$.

Biodegradable Bioharmonophore Functionalization. One milliliter of $1.5 \mathrm{mg} / \mathrm{mL}$ bioharmonophores was incubated with $1 \mathrm{mg}$ of Candida antarctica Lipase B (Sigma) for $2 \mathrm{~h}$, which hydrolyzes the PLLA polymer to increase the number of carboxyl groups. The bioharmonophore suspension was centrifuged at $13500 \mathrm{rpm}$ for $3 \mathrm{~min}$ and resuspended in $1 \%$ Tween 80 in $1 \mathrm{X}$ PBS and mixed with $10 \mathrm{mg}$ of $\mathrm{N}$-(3-(dimethylamino)propyl)- $\mathrm{N}^{\prime}$-ethylcarbodiimide hydrochloride (EDC) (Sigma), $10 \mathrm{mg}$ of $N$-hydroxysuccinimide (NHS) (Sigma), and $10 \mathrm{mg}$ of methoxypolyethylene glycol amine $5000 \mathrm{Da}$ (mPEG Amine) (Sigma) for $2 \mathrm{~h}$. The suspension was centrifuged and resuspended in $1 \%$ Tween 80 in $1 \mathrm{X}$ PBS and stored at $4{ }^{\circ} \mathrm{C}$ prior to use.

For further functionalization experiments thiol-PEG-amine 2000 $\mathrm{Da}\left(\mathrm{SH}-\mathrm{PEG}-\mathrm{NH}_{2}\right)$ (Sigma) was used as a platform for bioorthogonal click chemistry. In a similar setup, $10 \mathrm{mg}$ of EDC, $10 \mathrm{mg}$ of NHS, and $10 \mathrm{mg}$ of thiol-PEG- $\mathrm{NH}_{2}$ were incubated for $2 \mathrm{~h}$. The suspension was centrifuged and resuspended in $1 \%$ Tween 80 in 1X PBS with methyltetrazine-PEG4-maleimide (Click Chemistry Tools) of $200 \mu \mathrm{M}$ final concentration. The mixture was incubated for $2 \mathrm{~h}$ at room temperature, centrifuged, and resuspended in $1 \%$ Tween 80 in $1 \mathrm{X}$ PBS.

The other click chemistry pair trans-cyclooctene (TCO)-PEG3maleimide (Click Chemistry Tools) ( $3 \mathrm{mM}$ in $200 \mu \mathrm{L}$ of PBS) was incubated for $2 \mathrm{~h}$ with cysteine-containing Tat or P32 targeting peptides ( $1 \mathrm{mM}$ final concentration) depending on the application. The peptides were passed through Illustra Microspin G25 columns (GE Healthcare) to remove TCO-PEG3-maleimide.

A $200 \mu$ Lportion of tetrazine-modified bioharmonophores was incubated with $20 \mu \mathrm{L}$ of TCO-modified peptide for $2 \mathrm{~h}$. The bioharmonophore suspension was washed with $1 \%$ Tween 80 in $1 \mathrm{X}$ PBS to remove unbound peptides and resuspended in $1 \mathrm{X}$ PBS to be immediately used for cell culture experiments.

Cellular Degradation and Toxicity. MDA-MB-435-DsRed cancer cells were kindly gifted by Prof. R. Klemke. The cells were cultured at $37{ }^{\circ} \mathrm{C}, 5 \% \mathrm{CO}_{2}$, in high glucose DMEM with GlutaMAX (10569010, Thermo Fisher), supplemented with 10\% FBS (P4037500, Pan Biotech) and 1X penicillin-streptomycin solution (15140122, Thermo Fisher). The cells were cultured on six-well plates (140675, Thermo Fisher) until they reached $\sim 80 \%$ confluency and were incubated with $400 \mu \mathrm{L}$ Tat-derived cell penetrating peptide coated bioharmonophores overnight. The cells were washed with $1 \mathrm{X}$ PBS twice and detached using 0.05\% Trypsin-EDTA (25300054, Thermo Fisher) in order to remove bioharmonophores that did not enter the cancer cells. Detached cells were centrifuged for $5 \mathrm{~min}$ at $500 \mathrm{~g}$ to remove excess bioharmonophores that were not taken up, reseeded or ibiTreat-coated on eight-well slides (80826, Ibidi $\mathrm{GmbH}$ ), and fixed after 6, 30, 54, and $78 \mathrm{~h}$ to monitor bioharmonophores degradation. The samples were then washed three times with $1 \mathrm{X}$ PBS and stained with the CellMask Orange Membrane dye (Invitrogen). The samples were washed again and imaged subsequently. To determine cell viability after treatment with functionalized bioharmonophores, trypan blue exclusion method was used. Briefly, cells in triplicates seeded in 96-well tissue culture plates (167008, Thermo Fisher) were exposed to varying concentrations of functionalized bioharmonophores for 48 or $72 \mathrm{~h}$. After incubation, cells were washed with $1 \mathrm{X}$ PBS twice and detached as described above. Ten microliters of the cell suspension was then mixed with 10 $\mu \mathrm{L}$ of $0.4 \%$ Trypan Blue, and $4 \mu \mathrm{L}$ of this mixture was added to the cell-counting slide (C10228, Thermo Fisher) and measured using Countess II Automated cell counter (Thermo Fisher). The viability was expressed as a fold difference of the untreated samples for each time point.

Subcellular Localization of Bioharmonophores upon Cellular Uptake. HeLa cells were cultured at $37{ }^{\circ} \mathrm{C}, 5 \% \mathrm{CO}_{2}$, in high glucose DMEM with GlutaMAX (10569010, Thermo Fisher), supplemented with 10\% FBS (P40-37500, Pan Biotech) and 1X penicillin-streptomycin solution (15140122, Thermo Fisher). A 13 $\mathrm{mm}$ round coverslip was coated with $0.1 \%$ gelatin (G1393, Merck) for $1 \mathrm{~h}$, and cells were seeded to reach around $70 \%$ confluency. The following day, $400 \mu \mathrm{L}$ of freshly prepared thiol-PEG functionalized bioharmonophores ${ }^{46,47}(2 \mathrm{mg} / \mathrm{mL})$ or an equal volume of $1 \mathrm{X}$ PBS (control) was added to the cells and incubated for $24 \mathrm{~h}$. The cells were washed with 1X DPBS (containing $\mathrm{Ca}^{2+}$ and $\mathrm{Mg}^{2+}, 14040133$, Gibco) to remove the excess of not internalized bioharmonophores, fixed with 4\% PFA for 15 min, permeabilized with 1X PBST (1X PBS containing $0.01 \%$ Tween 20 ) for $15 \mathrm{~min}$, blocked with $10 \%$ goat serum in $1 \mathrm{X}$ PBST for at least $1 \mathrm{~h}$, and incubated overnight with mouse antiLAMP2 (ab25631, Abcam, 1:200) in 5\% goat serum at $4{ }^{\circ} \mathrm{C}$. The next day, cells were rinsed with $1 \mathrm{X}$ PBST and incubated for $1 \mathrm{~h}$ with the goat antimouse Alexa488 conjugated secondary antibody (A28175, Thermo Scientific, 1:1000), washed thoroughly with $1 \mathrm{X}$ PBST, and mounted in mowiol mounting medium containing $2.5 \% \mathrm{v} / \mathrm{v}$ DABCO (290734, Merck).

Toxicity Assay and Thioflavin T Staining. For toxicity assay, cells were grown in 96-well plates and were incubated with bioharmonophores at different concentrations for 48 and $72 \mathrm{~h}$. After the incubation period, the cells were detached with trypsinization, and their viability was analyzed using Trypan Blue (Sigma) staining.

For thioflavin staining, cells were seeded in an eight-well chamber (ibidi) with $50 \%$ confluency. The cells were treated with either Amyloid Beta Peptide (Bachem) or $5 \mu \mathrm{L}$ of bioharmonophores for 24 $h$ and extensively washed with $1 X$ PBS to remove excess peptides and bioharmonophores. To evaluate whether bioharmonophores induce fibril formation the cells were fixed with $4 \%$ PFA for $10 \mathrm{~min}$ and washed with $1 \mathrm{X}$ PBS three times. Afterward, $0.05 \%$ Thioflavin T (Sigma) solution was added to the sample for $8 \mathrm{~min}$, and excess dye was washed with $80 \%$ ethanol for $5 \mathrm{~min}$. The washing step was repeated three times, and the samples were imaged using confocal microscopy.

Zebrafish Cancer Model and Bioharmonophore Targeting. Animal experiments and zebrafish husbandry were approved by the "Kantonales Veterinaeramt Basel-Stadt". MDA-MB-435-DsRed cancer cells were injected into the Duct of Cuvier of $T g(f l i 1: e g f p)$ zebrafish embryos at 2 days post fertilization (dpf). After injection, embryos were incubated for $1 \mathrm{~h}$ at $29^{\circ} \mathrm{C}$ for recovery and cell transfer then verified by fluorescence microscopy. Fish harboring red cells were incubated at $35^{\circ} \mathrm{C}$ essentially as described before. ${ }^{29,30}$ Fish were anaesthetized and embedded in low melting agarose as described previously ${ }^{48}$ and were imaged at $5 \mathrm{dpf}$ for assessing cancer cell localization.

For targeting experiments, p32/gC1qR ligand-functionalized bioharmonophores were injected into the zebrafish embryos $24 \mathrm{~h}$ after cancer cell injection following the same procedure. In vivo bioharmonophores targeting was evaluated at $5 \mathrm{dpf}$ using nonlinear laser scanning microscopy.

Transmission Electron Microscopy. Bioharmonophore samples were spun down to remove aggregated nanoparticles at $3000 \mathrm{rpm}$ for $3 \mathrm{~min}$. Five microliters of the sample (i.e., the supernatant of the centrifuged solution) was placed on a carbon coated grid (Quantifoil, D) previously glow-discharged for $30 \mathrm{~s}$ (Emitech K100X, GB). The drop was allowed to remain for $60 \mathrm{~s}$; after this interval, excess fluid was drained along the periphery using a piece of filter paper followed by staining with $2 \%$ uranyl acetate for 1 and 15 s, respectively. Excess moisture was drained after each step, and when dry, the grid was examined in an FEI Morgagni 268 TEM operated at $100 \mathrm{kV}$.

Nonlinear and Confocal Laser Scanning Microscopy. Bioharmonophores were immobilized in low melting agarose by mixing $200 \mu \mathrm{L}$ of bioharmonophore with $100 \mu \mathrm{L}$ of $1 \%$ SeaPlaque low melting agarose (Lonza) solution in eight-well imaging chambers 
(Lab-Tek). Imaging experiments were performed either on a Zeiss LSM 780 microscope (Carl Zeiss AG) equipped with a spectral GaAsP detector and a tunable two-photon laser source (Chameleon Ultra II, Coherent, Inc.) using an LD C-Apochromat $40 \times / 1.1$ water immersion objective lens (Carl Zeiss AG) or on a Leica SP5 microscope equipped with 2 Hybrid Detectors (HyD, Leica) and a tunable two-photon laser source (Mai-Tai 690-1020, Spectraphysics), using a HC PL Apochromat $63 \times / 1.40$ Oil immersion objective lens (Leica). Throughout the imaging experiments, bioharmonophores were illuminated with $850 \mathrm{~nm}$ incident wavelength, and the SHG signal was collected between 405 and $435 \mathrm{~nm}$ or with a GaAsP spectral wavelength detector for spectral measurements.

Statistical Analysis. All numerical values represent mean \pm s.d. Sample sizes $(n)$ are given in the figure legends for each experiment. Each experiment was repeated at least three times. Normal distribution of data sets was established using the D'Agostino \& Pearson omnibus normality test where $P>0.05$ indicated Gaussian distribution. When all of the data sets had Gaussian distribution, oneway Anova was used for multiple comparisons followed by Tukey's multiple comparisons. When one or more data sets showed a nonGaussian distribution or high degree of variance as in the case of zebrafish tumor models, a Kruskal-Wallis test was applied along with Dunn's multiple comparisons. For all statistical tests, a $P$ value was reported, n.s., $P>0.05, *, P<0.05$, **, $P<0.005$, ***, $P<0.001$, ****, $P<0.0001$. Second-order polynomial fit and one phase exponential decay values were calculated, and graphs were drawn using GraphPad Prism 6.

\section{ASSOCIATED CONTENT}

\section{(s) Supporting Information}

The Supporting Information is available free of charge at https://pubs.acs.org/doi/10.1021/acsnano.0c10634.

Supplementary figures as described in the text (Figures 1-20) and supplementary notes describing optical characterization of bioharmonophores and determination of the fraction of bioharmonophore-labeled tumors in a zebrafish cancer model (PDF)

\section{AUTHOR INFORMATION}

\section{Corresponding Author}

Periklis Pantazis - Department of Biosystems Science and Engineering (D-BSSE), Eidgenössische Technische Hochschule (ETH) Zurich, 4058 Basel, Switzerland; Department of Bioengineering, Imperial College London, London SW7 2AZ, U.K.; (1) orcid.org/0000-0002-83679332; Email: p.pantazis@imperial.ac.uk

\section{Authors}

Ali Yasin Sonay - Department of Biosystems Science and Engineering (D-BSSE), Eidgenössische Technische Hochschule (ETH) Zurich, 4058 Basel, Switzerland

Konstantinos Kalyviotis - Department of Bioengineering, Imperial College London, London SW7 2AZ, U.K.

Sine Yaganoglu - Department of Biosystems Science and Engineering (D-BSSE), Eidgenössische Technische Hochschule (ETH) Zurich, 4058 Basel, Switzerland

Aysen Unsal - Department of Bioengineering, Imperial College London, London SW7 2AZ, U.K.

Martina Konantz - Department of Biomedicine, University Hospital Basel and University of Basel, 4031 Basel, Switzerland

Claire Teulon - Laboratory for Fundamental BioPhotonics, Institute of Bioengineering, School of Engineering, Ecole Polytechnique Fédérale de Lausanne, 1015 Lausanne, Switzerland
Ingo Lieberwirth - Max Planck Institute for Polymer Research, 55128 Mainz, Germany; 이 orcid.org/0000-00031323-524X

Sandro Sieber - Division of Pharmaceutical Technology, Department of Pharmaceutical Sciences, University of Basel, 4031 Basel, Switzerland

Shuai Jiang - Max Planck Institute for Polymer Research, 55128 Mainz, Germany; 다 orcid.org/0000-0002-86393495

Shahed Behzadi - Max Planck Institute for Polymer Research, 55128 Mainz, Germany

Daniel Crespy - Max Planck Institute for Polymer Research, 55128 Mainz, Germany; Department of Materials Science and Engineering, School of Molecular Science and Engineering, Vidyasirimedhi Institute of Science and Technology (VISTEC), Rayong 21210, Thailand

Katharina Landfester - Max Planck Institute for Polymer Research, 55128 Mainz, Germany

Sylvie Roke - Laboratory for Fundamental BioPhotonics, Institute of Bioengineering, School of Engineering, Institute of Materials Science and Engineering, School of Engineering, and Lausanne Centre for Ultrafast Science, Ecole Polytechnique Fédérale de Lausanne, 1015 Lausanne, Switzerland; (1) orcid.org/0000-0002-6062-7871

Claudia Lengerke - Department of Biomedicine, University Hospital Basel and University of Basel, 4031 Basel, Switzerland; Division of Hematology, University Hospital Basel, 4031 Basel, Switzerland

Complete contact information is available at: https://pubs.acs.org/10.1021/acsnano.0c10634

\section{Author Contributions}

A.Y.S. conceived and A.Y.S. and P.P refined the idea. A.Y.S produced and characterized bioharmonophores with the help of K.K., A.U., I.L., S.J., S.B., D.C., and K.L. A.Y.S., C.T., and S.R. performed optical characterization. K.K., S.Y., A.U., and A.Y.S. performed cell culture experiments. A.Y.S., S.Y., and K.K. generated in vitro and in vivo imaging data. M.K. and S.S. generated the zebrafish cancer model and performed bioharmonophore injections with the help of C.L.. A.Y.S. and P.P. wrote the manuscript and all authors contributed to editing the manuscript. P.P. supervised the project.

\section{Notes}

The authors declare the following competing financial interest(s): A patent application has been filed relating to aspects of the work described in this manuscript. Authors listed on the patent: P.P., A.Y.S., K.L., and D.C.

This publication was previously submitted to a preprint server: Sonay, A. Y.; Yaganoglu, S.; Konantz, M.; Teulon, C.; Sieber, S.; Jiang, S.; Behzadi, S.; Crespy, D.; Landfester, K.; Roke, S.; Langerke, C.; Pantazis, P. Biodegradable Harmonophores for Targeted High-Resolution In Vivo Tumor Imaging. bioRxiv 2019, 694760, https://www.biorxiv.org/content/10.1101/ 694760v1 (accessed 2021-02-02).

\section{ACKNOWLEDGMENTS}

We thank members of the Pantazis group for discussion and feedback. We thank W. P. Dempsey for feedback on the manuscript. We thank the Scientific Center for Optical and Electron Microscopy (ScopeM) for their help in imaging bioharmonophores. We also thank T. Weber of the Crystallography Laboratory of ETH Zurich for his help with 
the XRD analysis. We thank R. Klemke for kindly providing the MDA-MB-435-DsRed cell line. We thank M. Affolter and H. G. Belting for providing some of the zebrafish eggs. The Facility for Imaging by Light Microscopy (FILM) at Imperial College London is partially supported by funding from the Wellcome Trust (Grant No. 104931/Z/14/Z). This work was supported by the Swiss National Science Foundation (SNF Grant No. 31003A_144048), the European Union Seventh Framework Program (Marie Curie Career Integration Grant (CIG) No. 334552), and the Swiss National Center of Competence in Research (NCCR) "Nanoscale Science", which were awarded to P.P. who is a Royal Society Wolfson Research Merit Award holder.

\section{REFERENCES}

(1) Luker, G. D.; Luker, K. E. Optical Imaging: Current Applications and Future Directions. J. Nucl. Med. 2007, 49 (1), 1-4.

(2) Lindner, J. R.; Link, J. Molecular Imaging in Drug Discovery and Development. Circulation: Cardiovascular Imaging 2018, DOI: 10.1161/CIRCIMAGING.117.005355.

(3) Koch, M.; Ntziachristos, V. Advancing Surgical Vision with Fluorescence Imaging. Annu. Rev. Med. 2016, 67 (1), 153-164.

(4) Lamberts, L. E.; Koch, M.; de Jong, J. S.; Adams, A. L. L.; Glatz, J.; Kranendonk, M. E. G.; Terwisscha van Scheltinga, A. G. T.; Jansen, L.; de Vries, J.; Lub-de Hooge, M. N.; Schröder, C. P.; Jorritsma-Smit, A.; Linssen, M. D.; de Boer, E.; van der Vegt, B.; Nagengast, W. B.; Elias, S. G.; Oliveira, S.; Witkamp, A. J.; Mali, W. P. T. M.; et al. Tumor-Specific Uptake of Fluorescent Bevacizumab-IRDye800CW Microdosing in Patients with Primary Breast Cancer: A Phase I Feasibility Study. Clin. Cancer Res. 2017, 23 (11), 2730-2741.

(5) Billinton, N.; Knight, A. W. Seeing the Wood through the Trees: A Review of Techniques for Distinguishing Green Fluorescent Protein from Endogenous Autofluorescence. Anal. Biochem. 2001, 291 (2), 175-197.

(6) Pantazis, P.; Maloney, J.; Wu, D.; Fraser, S. E. Second Harmonic Generating (SHG) Nanoprobes for in Vivo Imaging. Proc. Natl. Acad. Sci. U. S. A. 2010, 107 (33), 14535-14540.

(7) Rogov, A.; Mugnier, Y.; Bonacina, L. Harmonic Nanoparticles: Noncentrosymmetric Metal Oxides for Nonlinear Optics. J. Opt. 2015, 17 (3), 033001.

(8) Extermann, J.; Bonacina, L.; Cuña, E.; Kasparian, C.; Mugnier, Y.; Feurer, T.; Wolf, J.-P. Nanodoublers as Deep Imaging Markers for Multi-Photon Microscopy. Opt. Express 2009, 17 (17), 15342-15349.

(9) Dubreil, L.; Leroux, I.; Ledevin, M.; Schleder, C.; Lagalice, L.; Lovo, C.; Fleurisson, R.; Passemard, S.; Kilin, V.; Gerber-Lemaire, S.; Colle, M.-A.; Bonacina, L.; Rouger, K. Multi-Harmonic Imaging in the Second Near-Infrared Window of Nanoparticle-Labeled Stem Cells as a Monitoring Tool in Tissue Depth. ACS Nano 2017, 11 (7), 66726681.

(10) Dempsey, W. P.; Fraser, S. E.; Pantazis, P. SHG Nanoprobes: Advancing Harmonic Imaging in Biology. BioEssays 2012, 34 (5), 351-360.

(11) Magouroux, T.; Extermann, J.; Hoffmann, P.; Mugnier, Y.; Le Dantec, R.; Jaconi, M. E.; Kasparian, C.; Ciepielewski, D.; Bonacina, L.; Wolf, J.-P. High-Speed Tracking of Murine Cardiac Stem Cells by Harmonic Nanodoublers. Small 2012, 8 (17), 2752-2756.

(12) Culic-Viskota, J.; Dempsey, W. P; Fraser, S. E; Pantazis, P. Surface Functionalization of Barium Titanate SHG Nanoprobes for in Vivo Imaging in Zebrafish. Nat. Protoc. 2012, 7 (9), 1618-1633.

(13) Sugiyama, N.; Sonay, A. Y.; Tussiwand, R.; Cohen, B. E.; Pantazis, P. Effective Labeling of Primary Somatic Stem Cells with $\mathrm{BaTiO}_{3}$ Nanocrystals for Second Harmonic Generation Imaging. Small 2018, 14 (8), 1703386.

(14) Staedler, D.; Magouroux, T.; Hadji, R.; Joulaud, C.; Extermann, J.; Schwung, S.; Passemard, S.; Kasparian, C.; Clarke, G.; Gerrmann, M.; Le Dantec, R.; Mugnier, Y.; Rytz, D.; Ciepielewski, D.; Galez, C.; Gerber-Lemaire, S.; Juillerat-Jeanneret, L.; Bonacina, L.; Wolf, J.-P.
Harmonic Nanocrystals for Biolabeling: A Survey of Optical Properties and Biocompatibility. ACS Nano 2012, 6 (3), 2542-2549.

(15) Lakshmanan, A.; Zhang, S.; Hauser, C. A. E. Short SelfAssembling Peptides as Building Blocks for Modern Nanodevices. Trends Biotechnol. 2012, 30 (3), 155-165.

(16) Kholkin, A.; Amdursky, N.; Bdikin, I.; Gazit, E.; Rosenman, G. Strong Piezoelectricity in Bioinspired Peptide Nanotubes. ACS Nano 2010, 4 (2), 610-614.

(17) Handelman, A.; Beker, P.; Amdursky, N.; Rosenman, G. Physics and Engineering of Peptide Supramolecular Nanostructures. Phys. Chem. Chem. Phys. 2012, 14 (18), 6391-6408.

(18) Staff, R. H.; Schaeffel, D.; Turshatov, A.; Donadio, D.; Butt, H.J.; Landfester, K.; Koynov, K.; Crespy, D. Particle Formation in the Emulsion-Solvent Evaporation Process. Small 2013, 9 (20), 35143522.

(19) Rabotyagova, O. S.; Cebe, P.; Kaplan, D. L. Role of Polyalanine Domains in $\beta$-Sheet Formation in Spider Silk Block Copolymers. Macromol. Biosci. 2010, 10 (1), 49-59.

(20) Handelman, A.; Kuritz, N.; Natan, A.; Rosenman, G. Reconstructive Phase Transition in Ultrashort Peptide Nanostructures and Induced Visible Photoluminescence. Langmuir 2016, 32 (12), $2847-2862$

(21) Handelman, A.; Lavrov, S.; Kudryavtsev, A.; Khatchatouriants, A.; Rosenberg, Y.; Mishina, E.; Rosenman, G. Nonlinear Optical Bioinspired Peptide Nanostructures. Adv. Opt. Mater. 2013, 1 (11), 875-884.

(22) de Beer, A. G. F.; de Aguiar, H. B.; Nijsen, J. F. W.; Roke, S. Detection of Buried Microstructures by Nonlinear Light Scattering Spectroscopy. Phys. Rev. Lett. 2009, 102 (9), 095502.

(23) Roke, S.; Gonella, G. Nonlinear Light Scattering and Spectroscopy of Particles and Droplets in Liquids. Annu. Rev. Phys. Chem. 2012, 63 (1), 353-378.

(24) Tsuji, H.; Ogiwara, M.; Saha, S. K.; Sakaki, T. Enzymatic, Alkaline, and Autocatalytic Degradation of Poly-L-Lactic Acid: Effects of Biaxial Orientation. Biomacromolecules 2006, 7 (1), 380-387.

(25) Sepp, R.; Szabo, I.; Uda, H.; Sakamoto, H. Rapid Techniques for DNA Extraction from Routinely Processed Archival Tissue for Use in PCR. J. Clin. Pathol. 1994, 47 (4), 318-323.

(26) Lewin, M.; Carlesso, N.; Tung, C.-H.; Tang, X.-W.; Cory, D.; Scadden, D. T.; Weissleder, R. Tat Peptide-Derivatized Magnetic Nanoparticles Allow in Vivo Tracking and Recovery of Progenitor Cells. Nat. Biotechnol. 2000, 18 (4), 410-414.

(27) Nguyen, H.; Gitig, D. M.; Koff, A. Cell-Free Degradation of p27 kip1, a G1 Cyclin-Dependent Kinase Inhibitor, Is Dependent on CDK2 Activity and the Proteasome. Mol. Cell. Biol. 1999, 19 (2), $1190-1201$

(28) Lee, H.-J.; Shin, S. Y.; Choi, C.; Lee, Y. H.; Lee, S.-J. Formation and Removal of Alpha-Synuclein Aggregates in Cells Exposed to Mitochondrial Inhibitors. J. Biol. Chem. 2002, 277 (7), 5411-5417.

(29) Konantz, M.; Balci, T. B.; Hartwig, U. F.; Dellaire, G.; André, M. C.; Berman, J. N.; Lengerke, C. Zebrafish Xenografts as a Tool for in Vivo Studies on Human Cancer. Ann. N. Y. Acad. Sci. 2012, 1266 (1), 124-137.

(30) Stoletov, K.; Kato, H.; Zardouzian, E.; Kelber, J.; Yang, J.; Shattil, S.; Klemke, R. Visualizing Extravasation Dynamics of Metastatic Tumor Cells. J. Cell Sci. 2010, 123 (13), 2332-2341.

(31) Stoletov, K.; Montel, V.; Lester, R. D.; Gonias, S. L.; Klemke, R. High-Resolution Imaging of the Dynamic Tumor Cell-Vascular Interface in Transparent Zebrafish. Proc. Natl. Acad. Sci. U. S. A. 2007, 104 (44), 17406-17411.

(32) Agemy, L.; Kotamraju, V. R.; Friedmann-Morvinski, D.; Sharma, S.; Sugahara, K. N.; Ruoslahti, E. Proapoptotic PeptideMediated Cancer Therapy Targeted to Cell Surface p32. Mol. Ther. 2013, 21 (12), 2195-2204.

(33) LeBert, D. C.; Squirrell, J. M.; Huttenlocher, A.; Eliceiri, K. W. Second Harmonic Generation Microscopy in Zebrafish. Methods Cell Biol. 2016, 133, 55-68.

(34) Nakamura, Y.; Mochida, A.; Choyke, P. L.; Kobayashi, H. Nanodrug Delivery: Is the Enhanced Permeability and Retention 
Effect Sufficient for Curing Cancer? Bioconjugate Chem. 2016, 27 (10), 2225-2238.

(35) Jain, R. K.; Stylianopoulos, T. Delivering Nanomedicine to Solid Tumors. Nat. Rev. Clin. Oncol. 2010, 7 (11), 653-664.

(36) Schädlich, A.; Caysa, H.; Mueller, T.; Tenambergen, F.; Rose, C.; Göpferich, A.; Kuntsche, J.; Mäder, K. Tumor Accumulation of NIR Fluorescent PEG-PLA Nanoparticles: Impact of Particle Size and Human Xenograft Tumor Model. ACS Nano 2011, 5 (11), 87108720.

(37) Perry, J. L.; Reuter, K. G.; Luft, J. C.; Pecot, C. V.; Zamboni, W.; DeSimone, J. M. Mediating Passive Tumor Accumulation through Particle Size, Tumor Type, and Location. Nano Lett. 2017, 17 (5), 2879-2886.

(38) Zhou, Z.; Lu, Z.-R. Molecular Imaging of the Tumor Microenvironment. Adv. Drug Delivery Rev. 2017, 113, 24-48.

(39) König, K.; Ehlers, A.; Riemann, I.; Schenkl, S.; Bückle, R.; Kaatz, M. Clinical Two-Photon Microendoscopy. Microsc. Res. Tech. 2007, 70 (5), 398-402.

(40) Sanchez, G. N.; Sinha, S.; Liske, H.; Chen, X.; Nguyen, V.; Delp, S. L.; Schnitzer, M. J. In Vivo Imaging of Human Sarcomere Twitch Dynamics in Individual Motor Units. Neuron 2015, 88 (6), $1109-1120$.

(41) Yu, Z.; Pestell, T. G.; Lisanti, M. P.; Pestell, R. G. Cancer Stem Cells. Int. J. Biochem. Cell Biol. 2012, 44 (12), 2144-51.

(42) Kachynski, A. V.; Pliss, A.; Kuzmin, A. N.; Ohulchanskyy, T. Y.; Baev, A.; Qu, J.; Prasad, P. N. Photodynamic Therapy by in Situ Nonlinear Photon Conversion. Nat. Photonics 2014, 8, 455.

(43) Costa, D. F.; Mendes, L. P.; Torchilin, V. P. The Effect of Lowand High-Penetration Light on Localized Cancer Therapy. Adv. Drug Delivery Rev. 2019, 138, 105.

(44) Adler-Abramovich, L.; Gazit, E. The Physical Properties of Supramolecular Peptide Assemblies: From Building Block Association to Technological Applications. Chem. Soc. Rev. 2014, 43 (20), 68816893.

(45) Reches, M.; Gazit, E. Controlled Patterning of Aligned SelfAssembled Peptide Nanotubes. Nat. Nanotechnol. 2006, 1 (3), 195200.

(46) Loretz, B.; Thaler, M.; Bernkop-Schnürch, A. Role of Sulfhydryl Groups in Transfection? A Case Study with Chitosan-NAC Nanoparticles. Bioconjugate Chem. 2007, 18 (4), 1028-35.

(47) Gasparini, G.; Sargsyan, G.; Bang, E. K.; Sakai, N.; Matile, S. Ring Tension Applied to Thiol-Mediated Cellular Uptake. Angew. Chem., Int. Ed. 2015, 54 (25), 7328-31.

(48) Dempsey, W. P.; Fraser, S. E.; Pantazis, P. PhOTO Zebrafish: A Transgenic Resource for in Vivo Lineage Tracing during Development and Regeneration. PLoS One 2012, 7 (3), No. e32888. 